\title{
Study on Protection of HTS Coil Against Quench Due to Temperature Rise of Long Part of HTS Wires
}

\author{
Takuya Minagawa, Yasutaka Fujimoto, Osami Tsukamoto
}

\begin{abstract}
Generally, HTS coated conductor (CC) are hard to be quenched by local and transient disturbances during normal operation because of high temperature margin and high heat capacity of the conductor. However, the CCs still have possibilities of unexpected quenches originated by appearance of local defects due to repeated mechanical stresses and by temperature rise of long part of the CCs due to malfunction of cryogenic system, for example. In this work, hot spot temperature of $\mathrm{CC}$ with copper stabilizer during quench protection sequence is calculated by a numerical analysis for quenches by both of the origins mentioned above and practical amount of the stabilizer is discussed not to over-protect but for a coil system to surely survive from sever damages caused by quenches considering quench origins.
\end{abstract}

Index Terms-Coated conductor, Quench protection, Hot spot temperature, Defect, Copper stabilizer.

\section{INTRODUCTION}

$\mathrm{R}$ ecently, R\&Ds of HTS motors and generators are progressing greatly and it is realized that sure quench protection of the HTS coils is essential for sustainable operation of these rotational machines. It is considered that there are three kinds of causes of unexpected quenches during operation of a coil, a) transient heat disturbances, b) appearance of non-recoverable local defects in the conductor which may be caused by fatigue effect due to repeated mechanical stresses during operation of the coil and c) temperature rise of long part of the conductor due to malfunction of cryogenic system, for example. HTS wires are hard to be quenched by the cause a) because of high temperature margin and high heat capacity of the conductor. However, the HTS coils still have possibilities to be quenched by the causes $b$ ) and c).

Most works on the quench issues are devoted to protection and stabilization of quenches by the cause a) [1] [5] and our previous works [6], [7] investigated the quenches by the cause

Manuscript received October 9, 2012. This research was (partially) supported by Japan Science and Technology Agency, JST, under Strategic Promotion of Innovative Research and Development Program.

Takuya Minagawa and Yasutaka Fujimoto are with Department of Electrical and Computer Engineering, Yokohama National University, 79-1 Tokiwadai, Hodogaya-ku, Yokohama 240-8501 JAPAN (Tel: +81-45-3394174; +81-45-339-4175; e-mail: minagawa@fujilab.dnj.ynu.ac.jp; fujimoto@ynu.ac.jp).

Osami Tsukamoto is a professor emeritus of Engineering, Yokohama National University, 79-1 Tokiwadai, Hodogayaku, Yokohama 240-8501 JAPAN(Tel:+81-45-339-4441; e-mail: osami-t@ynu.ac.jp).

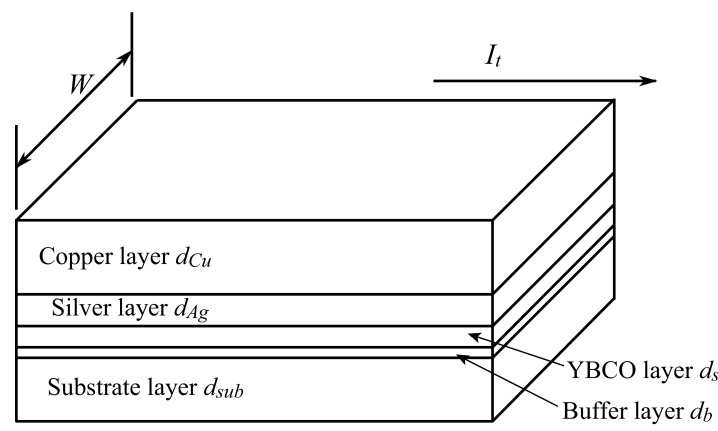

Fig. 1. Model CC with copper stabilizer.

b). However, protection against quenches by the cause c) is not well studied.

Generally, field coils of rotating machines are composed of multiple unit coils. When one of unit coils is quenched by the cause $b$ ), the unit coil cannot be reused and need to be replaced by a new unit coil. Considering this, the amount of stabilizer can be determined allowing additional damages in the coil caused by a quench. On the other hand, in a case that a coil is quenched by the cause c), the coil should recover superconducting stare after the quench by surely protecting the coil. In this case, amount of stabilizer should be determined to keep the hot spot temperature safe level not to cause damages in the coil. Considering the above there is a possibility to reduce amount of stabilizer compared with the case considering only protection against quench by local defects.

In this work, maximum temperature of copper stabilized CC during quench protection sequence (hot spot temperature: $T_{H S}$ ) is calculated for a case that temperature of long part of the $\mathrm{CC}$ exceeds current sharing temperature and the $\mathrm{CC}$ becomes resistive. Based on the calculation, necessary amount of the copper stabilizer is estimated to suppress $T_{H S}$ below a given threshold. These results are compared with the case of quench by the cause b).

\section{ANALYTICAL MODEL}

Necessary amount of copper stabilizer is investigated by calculating hot spot temperature $T_{H S}$ of a model CC.

\section{A. Structure of model CC}

A model conductor used in the analysis is illustrated in Fig. $1 . W$ is the width of the conductor and $d_{C u}, d_{A g}, d_{s}, d_{b}$, and $d_{\text {sub }}$ are thicknesses of the copper, silver, YBCO, buffer, and substrate layers, respectively. The copper stabilization layer is plated to the silver protection layer on the YBCO layer. 


\section{B. Calculation of time evolution of conductor temperature profile}

When the temperature of the conductor carrying transport current $I_{t}$ rises, the critical current of the superconducting layer $I_{\mathrm{c}}$ decreases and if $I_{c}$ becomes lower than $I_{t}$, sharing currents start to flow in the silver and copper layers. Current-sharing part of the conductor becomes resistive and dissipates heat. $I_{c}$ is given by the following equation as an approximation:

$$
I_{c}=\left\{\begin{array}{cc}
I_{c 0} \frac{T_{c}-T}{T_{c}-T_{o p}} & T_{o p} \leq T \leq T_{c} \\
0 & T>T_{c}
\end{array},\right.
$$

where $T$ and $T_{o p}$ are the conductor temperature and operation temperature of the coil respectively. $I_{c 0}$ is the critical current of the YBCO layer at $T_{o p}$. The critical temperature $T_{c}$ is assumed to be $91 \mathrm{~K}$ in the calculation. $T_{c}$ is dependent on magnetic field. We did the calculation putting $T_{c}=80 \mathrm{~K}$ and found that the results were not much affected by $T_{c}$. Assuming that distributions of currents in the silver, copper layers and superconducting layer are uniform in the cross sections of the corresponding layers, an equivalent circuit model for current distributions in layers of the CC can be formed as shown in the reference [6]. In the calculation it is assumed " $n$ " value of $\mathrm{CC}$ is high enough to assume that sharing currents in silver and copper layers $I_{s h}$ is 0 for $I_{t}<I_{c}$ and $\left(I_{t}-I_{c}\right)$ for $I_{t}>I_{c}$.

Knowing resistances of silver and copper layers, heat dissipation can be calculated in the current sharing part and using the following thermal equilibrium equation, time evolutions of conductor temperature profile can be numerically calculated.

$$
\frac{\partial}{\partial x}\left[K \frac{\partial T}{\partial x}\right]+P-W_{\text {cool }}=C_{\mathrm{p}} \frac{\partial T}{\partial t},
$$

where $K(\mathrm{~W} / \mathrm{m} \cdot \mathrm{K})$ and $C_{p}\left(\mathrm{~J} / \mathrm{m}^{3} \cdot \mathrm{K}\right)$ are the thermal conductivity and heat capacity per unit length of the conductor, respectively. $P(\mathrm{~W} / \mathrm{m})$ is joule heat per the unit length of the conductor. $K$ and $C_{p}$ are given by composition rule. $W_{\text {cool }}$ $(\mathrm{W} / \mathrm{m})$ is the heat conducted to and from the conductors of neighboring layers and put to zero in this analysis assuming the $\mathrm{CC}$ is thermally insulated. Details of the calculation are explained in the reference [6]. $T_{H S}$ is estimated from the calculated time evolutions of conductor temperature profile during sequence of quench detection and energy dump. The authors consider that the assumption of $W_{\text {cool }}=0$ is in safety side and reasonable for the purpose of the present analysis. Actually, necessary amount of $d_{C u}$ was calculated to suppress $T_{H S}$ below a certain level for a case of $W_{\text {cool }}=0$ [7] and a case that $W_{\text {cool }}$ was taken into account of the thermal conductivity of electric insulation layers among the neighboring conductors [6] and the calculation results were not much different.
TABLE I

DIMENSIONS OF MODEL YBCO CONDUCTOR

\begin{tabular}{ccc}
\hline \hline$W$ & Conductor width & $4.0 \times 10^{-3}$ \\
$d_{C u}$ & Copper layer thickness & Variable \\
$d_{A g}$ & Silver layer thickness & $5.0 \times 10^{-6}$ \\
$d_{s}$ & YBCO layer thickness & $\sim 1.0 \times 10^{-6}$ \\
$d_{b}$ & Buffer layer thickness & $0.1 \times 10^{-6}$ \\
$d_{s u b}$ & Substrate layer thickness & $50.0 \times 10^{-6}$ \\
\hline \hline
\end{tabular}

\section{Quench protection sequence}

It is assumed that the coil is protected by a commonly used sequence as follows. A quench in the coil is detected by monitoring the voltage across the resistive part of the conductor $V_{q}$ using a bridge balancing method. When $V_{q}$ exceeds a threshold level $V_{q s}$, the power supply is disconnected from the coil and stored energy of the coil is dumped to the dump resistor $R_{D}$. Usually, there is time delay $t_{d}$ to start energy dump after detection of quench. Decay of the coil conductor current $I_{t}(t)$ is given by $I_{t}=I_{t} 0^{\circ} \mathrm{e}^{t / \tau}$, where $I_{t 0}$ is the value of $I_{t}$ at $t=0$ when the energy dump starts and $\tau=$ $L_{c} / R_{D}$ is the energy dump time constant ( $L_{c}$ is the inductance of the coil). To protect the coil from a damage caused by a quench, the conductor and quench protection system should be designed so that $T_{H S}$ should not exceed a certain threshold.

When a coil conductor is composed of multiple elementary CCs and the conductor current $I_{m t}$ is equally distributed in the elementary CCs, the current of each of the elementary CCs $I_{t}$ is expressed as $I_{t}=I_{t} 0^{\circ} \mathrm{e}^{t / \tau}$ where $I_{t}=I_{m t} / N$ and $N$ is the number of elementary conductors composing the coil conductor. Then $T_{H S}$ of a coil wound of multiple elementary CCs can be estimated by estimating $T_{H S}$ of one elementary conductor.

\section{MODEL CONDUCTOR ANALYSIS}

Relations of $T_{H S}$ and amount of copper stabilizer $d_{C u}$ are investigated for a model conductor. Parameters of the model conductor are assumed as listed in Table I. Temperature dependencies of specific heats and resistivities of the conductor materials are taken into consideration in the calculation according to the data given in the reference [8].

In this analysis, $T_{o p}$ is determined to be $37 \mathrm{~K}$ assuming the coil is cooled by conduction cooling and considering the balance of efficiency of the cooling system and performance of CCs required for ship propulsion motors.

\section{A. Quench caused by local and non-recoverable defects}

Relations of $T_{H S}$ and $d_{C u}$ of the model conductor are obtained for the case that the model conductor carrying $I_{t 0}=\eta \cdot I_{c 0}$ is quenched by appearance of a local and nonrecoverable defect $(\eta:$ load factor). In the analysis it is assumed that the critical current of defected part of the conductor becomes zero and length of the defected part $L_{d}$ is $1 \mathrm{~mm}$. Fig. 2 shows $T_{H S}$ vs. $\tau$ in the case of $L_{d}=1 \mathrm{~mm}$ for $V_{q s}$ $=0.01$ and $0.1 \mathrm{~V}$ and for $d_{C u}=100 \sim 300 \mu \mathrm{m}$. Parameters used in the calculations and shown in Fig. 2 are the same in the following figures. Obviously from Fig. 2, $\tau$ should be small to 


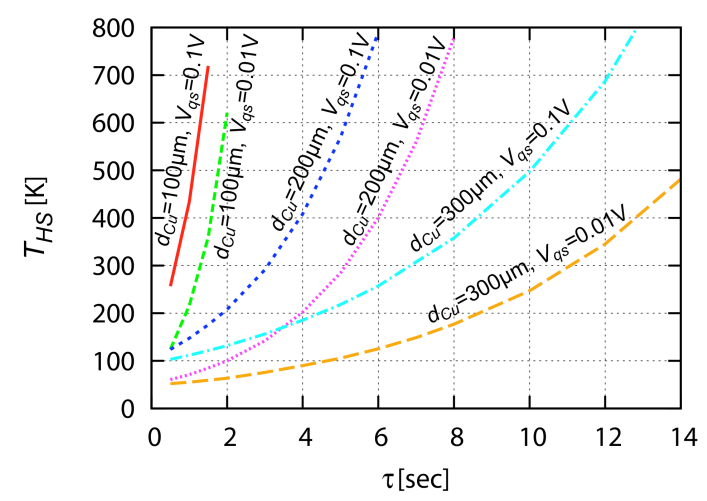

Fig. 2. Hot spot temperature $T_{H S}$ vs. quench dump time constant $\tau$. $\left(T_{o p}=37 \mathrm{~K}, I_{c 0}=306 \mathrm{~A}, I_{t 0}=200 \mathrm{~A}, L_{d}=10 \mathrm{~mm}\right)$.

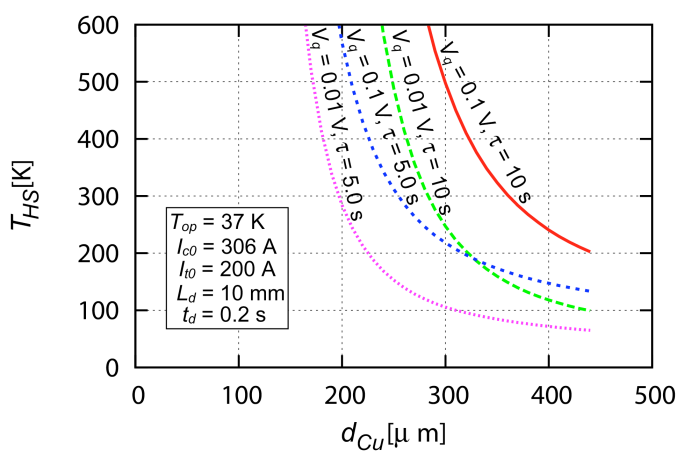

Fig. 3. $T_{H S}$ vs. $d_{c u}$ for $V_{q s}=0.01$ and $V_{q s}=0.1 \mathrm{~V}$.

suppress $T_{H S}$ and it is necessary to enhance electric insulation of a coil and increase coil conductor current to make $\tau$ small. It is also obvious that if $V_{q s}$ is reduced to $0.01 \mathrm{~V}, d_{C u}$ can be reduced. However, it should be pointed out that reduction of $V_{q s}$ increases possibility of miss detection of quench due to noise in quench detection signal. Fig. 3 (a) and (b) shows $T_{H S}$ vs. $d_{C u}$ for $V_{q s}=0.01$ and $0.1 \mathrm{~V}$. Fig. 4 is an example of time evolution of temperature profile of the model conductor in which defect of $L_{d}=1 \mathrm{~mm}$ appears at $x=0$ and $t=0 \mathrm{~s}$. At $t=259 \mathrm{~s} V_{q}$ reaches $V_{q s}=0.1 \mathrm{~V}$ and the quench dump sequence starts and at $t=284 \mathrm{~s}$ the temperature reaches $T_{H S}=$ $460 \mathrm{~K}$. In the above calculation delay time to start energy dump after detection of a quench $t_{d}$ is $0.2 \mathrm{~s}$. Calculation was done for $L_{d}=1 \sim 10 \mathrm{~mm}$ but values of $T_{H S}$ were not much different.

\section{B. Quench caused by temperature rise of long part of conductor}

When temperature of a coil increases, coil conductor becomes resistive in the range where $I_{t}$ exceeds $I_{c}$. When voltage across the resistive region $V_{q}$ exceeds $V_{q s}$, quench protection sequence starts. In the calculation it is assumed the conductor temperature distribution in the resistive region is uniform when a quench is detected. Fig. 5 (a) and (b) shows $T_{H S}$ vs. length of resistive part of the conductor $L$ for $\tau=5$ and $10 \mathrm{~s}$. As $L$ is longer, $T_{H S}$ is lower, because a quench is detected

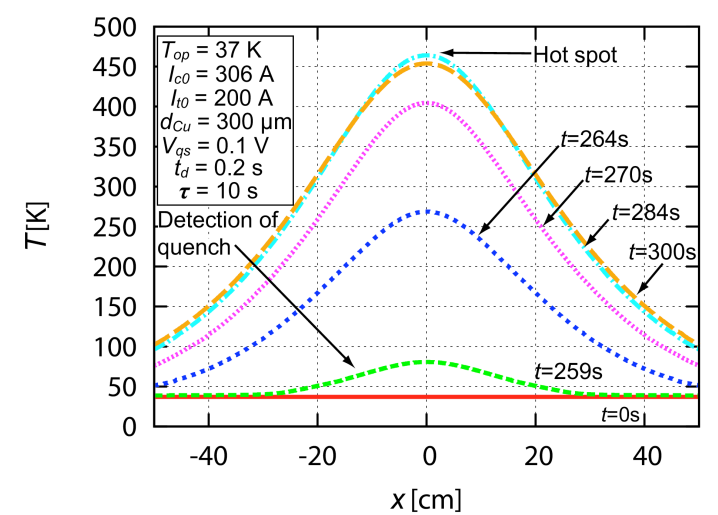

Fig. 4. Time evolution of conductor temperature profile for a $1 \mathrm{~mm}$ long defect.

at lower temperature rise of the conductor. As seen in Fig. 5, for $L$ exceeding a certain critical length $L_{0}$ which is dependent on the values of $d_{C u}$ and $\tau, T_{H S}$ becomes slightly above the current sharing temperature $T_{s h}$ at which $I_{t}$ equals to $I_{c}$. $T_{s h}$ and $L_{0}$ calculated using the parameters given in Fig. 5 are as follows. $T_{s h}=39.5 \mathrm{~K} . L_{0}=10.0,10.2,10.8 \mathrm{~m}$ for $d_{c u}=150$, 100, $50 \mu \mathrm{m}$, respectively in Fig. 5 (a) and $L_{0}=13.5,13.8$, $14.8 \mathrm{~m}$ for $d_{c u}=150,100,50 \mu \mathrm{m}$, respectively in Fig. 5 (b). The result shown in Fig. 5 suggests that if a coil cooling system can be so designed that $L$ is longer than $L_{0}$ in the worst fault case, $T_{H S}$ stays slightly above $T_{s h}$. For example, if $L$ is longer than $14.8 \mathrm{~m}$, a coil is safely protected from the quench even in the case of $d_{c u}=50 \mu \mathrm{m}$ and $\tau=10 \mathrm{~s}$. However, it should be pointed out that the criteria of $L>L_{0}$ is very critical. For example, in the case that $d_{c u}=50 \mu \mathrm{m}$ and $\tau=10 \mathrm{~s}, T_{H S}$ becomes well above $600 \mathrm{~K}$, if $L$ is slightly shorter than $L_{0}=$ $14.8 \mathrm{~m}$.

Fig. 6 shows an example of time evolution of temperature profile of the model conductor for a case that a part of the conductor of $L=13.0 \mathrm{~m}$ becomes resistive and the voltage across the resistive part exceeds $V_{q s}=0.1 \mathrm{~V}$. Energy dump sequence of $\tau=10 \mathrm{~s}$ starts at $t=0 \mathrm{~s}$ with delay of $t_{d}=0.2 \mathrm{~s}$. The conductor temperature becomes $T_{H S}=295 \mathrm{~K}$ at $t=40 \mathrm{~s}$.

\section{Case study of model coils}

Necessary amount of copper stabilizer is studied more details taking examples of model coils, Coil 1 and Coil 2 parameters of which are shown in Table II. Stored energy $W_{0}$ of the two coils $(2 \mathrm{MJ})$ and coil terminal voltage $V_{0}=R_{D} \cdot I_{t 0}$ $(2 \mathrm{kV})$ are the same at the beginning of the energy dump. Coil 1 is wound of single model conductor and Coil 2 of conductor assembled with two of the elementary model conductors in parallel. It is assumed that in Coil 2 currents in the two elementary conductors are uniform during the quench dump sequence. Operating conductor currents of Coils 1 and 2 $I_{t 0}$ are $200 \mathrm{~A}$ and $400 \mathrm{~A}$ and values of $\tau=2 W_{0} / R_{d} \cdot I_{t o}{ }^{2}$ are $10 \mathrm{~s}$ and $5 \mathrm{~s}$, respectively. $t_{d}$ is $0.2 \mathrm{~s}$. 


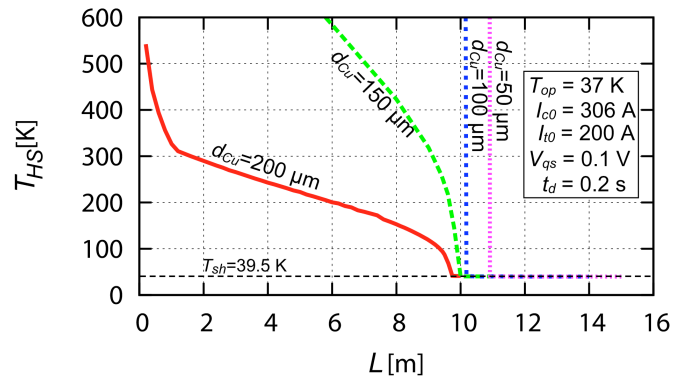

(a) $\tau=5.0 \mathrm{~s}$

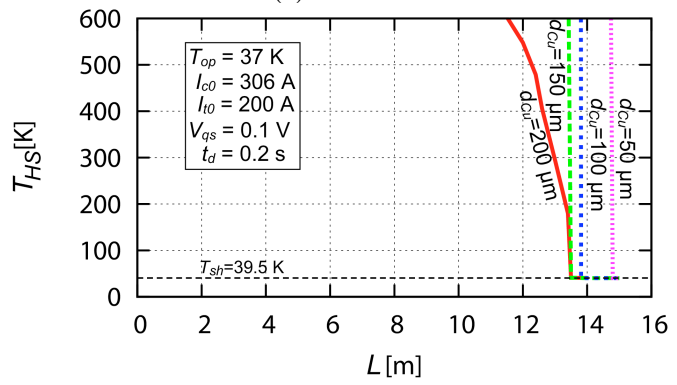

(b) $\tau=10 \mathrm{~s}$

Fig. 5. $T_{H S}$ vs. length of resistive part of the conductor $L$ for $\tau=5$ and $10 \mathrm{~s}$.

In Coil $1, d_{C u}$ should be more than $300 \mu \mathrm{m}$ to suppress $T_{H S}$ below $500 \mathrm{~K}$ at $V_{q s}=0.1 \mathrm{~V}$ for a quench caused by a local defect and if $d_{C u}$ is reduced to $200 \mu \mathrm{m}, T_{H S}$ becomes well over $1000 \mathrm{~K}$ and there is a risk to burn the coil even at more sensitive quench detection voltage $V_{q s}=0.01 \mathrm{~V}$ (See Fig. 2). However, in the case that a quench caused only by temperature rise of the conductor and that a quench is not caused by a local defect, $d_{C u}=200 \mu \mathrm{m}$ is enough to suppress $T_{H S}$ below $300 \mathrm{~K}$ at $V_{q s}=0.1 \mathrm{~V}$ if $L$ is longer than $13 \mathrm{~m}$. Moreover, if $L$ is longer than $14.8 \mathrm{~m}, d_{C u}$ can be reduced to $50 \mu \mathrm{m}$ (Fig. 5).

In Coil 2, $d_{C u}=200 \mu \mathrm{m}$ can suppress $T_{H S}$ below $600 \mathrm{~K}$ and the coil can get rid of a risk of burning at $V_{q s}=0.1 \mathrm{~V}$ for a quench caused by a local defect. In the case of a quench caused only by temperature rise of the conductor, $d_{C u}$ can be reduce to $50 \mu \mathrm{m}$ suppressing $T_{H S}$ slightly above $T_{s h}$ at $V_{q s}=$ $0.1 \mathrm{~V}$, if $L$ is longer than $10.8 \mathrm{~m}$.

\section{DISCUSSIONS AND CONCLUDING REMARKS}

Hot spot temperature of CCs $T_{H S}$ is investigated for case that a quench is caused by a local defect or by temperature rise in long part of CC. Calculation results are as follows:

1) It is shown that amount of $d_{C u}$ to suppress $T_{H S}$ below a given level for a quench caused by a local defect is larger than that for a quench caused by temperature rise of long part of coil conductor.

2) Considering that a coil cannot be reused when it is quenched by a non-recoverable defect, high value of $T_{H S}$ is allowable. Therefore, $d C u$ and $T_{H S}$ can be selected mainly considering protection from a quench caused by temperature rise of a long part of conductor. If $L$ is longer than $L_{0}$, value of $d_{C u}$ can be much reduced, while $T_{H S}$ stays slightly above $T_{s h}$.

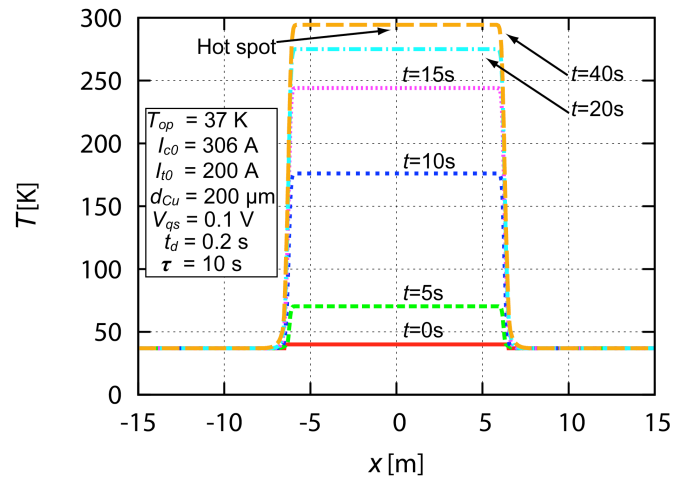

Fig. 6. Time evolution of conductor temperature profile for a $13 \mathrm{~m}$ long resistive region.

TABLE II

ASSUMED PARAMETER OF MODEL COIL

\begin{tabular}{c|c|c}
\hline \hline & Coil 1 & Coil 2 \\
\hline $\begin{array}{c}\text { Stored energy } \\
\text { at operations current }\end{array}$ & \multicolumn{2}{|c}{$2 \mathrm{MJ}$} \\
\hline $\begin{array}{c}\text { Coil terminal voltage at } \\
\text { the beginning of stored } \\
\text { energy dump }\left(I_{t} \times R_{D}\right)\end{array}$ & \multicolumn{2}{|c}{$2 \mathrm{kV}$} \\
\hline $\begin{array}{c}\text { Critical current of Coil } \\
\text { conductor at } T_{\text {op }}=37 \mathrm{~K}\end{array}$ & $306 \mathrm{~A}$ & $612 \mathrm{~A}$ \\
\hline $\begin{array}{c}\text { Operating coil conductor } \\
\text { current } I_{t 0}\end{array}$ & $\begin{array}{c}200 \mathrm{~A} \\
\text { (One model } \\
\text { conductor) }\end{array}$ & $\begin{array}{c}\text { (Two model conductors } \\
\text { are assembled) }\end{array}$ \\
\hline $\begin{array}{c}\text { Coil inductance } L_{c} \\
\text { Dump resistor } R_{D} \\
\text { Energy dump } \\
\text { time constant }\end{array}$ & $100 \mathrm{H}$ & $25 \mathrm{H}$ \\
$10 \Omega$ & $5 \Omega$ \\
\hline \hline
\end{tabular}

3) Increase of coil conductor current by assembling multiple CCs is effective to reduce $d_{C u}$ and increase the engineering current density as shown in Case 2.

Taking the above results into consideration, a coil of practically high level of current density of winding pack can be designed by investigating quench origins and reducing $d_{C u}$.

\section{REFERENCES}

[1] Y. Iwasa, L. Haigun, F. Jiarong, and B. Haid, "Quench and recovery of YBCO tape experimental and simulation results", IEEE Trans. Appl. Supercond. Vol. 13, No. 2, pp. 1772-1775, 2003.

[2] Y. Iwasa, J. Jankowski, S. Hahn, H. Lee, J. Bascunan, et al, "Stability and quench protection of coated YBCO "Composite" tape", IEEE Trans. Appl. Supercond. Vol. 15, No. 2, pp. 1683-1686, 2005.

[3] W. S. Kim, F. Trillaud, I. C. Ang, S. Y. Hahn, and Y. Iwasa, "Normal Zone propagation in YBCO Winding Pack Models", IEEE Trans. Appl. Supercond. Vol. 17, No. 2, pp. 2478-2481, 2007.

[4] J. Pelegrin, E. Martinez, L. A. Angurel, X. Yi-Yuan, and V. Selvamanickam, "Numerical and Experimental Analysis of Normal Zone Propagaton on 2G HTS Wires", IEEE Trans. Appl. Supercond. Vol. 21. No. 3, pp. 3041-3044, 2011.

[5] D. G. Yang, J. B. Song, Y.H. Choi, M.C. Ahn, J.B. Na, et al, "Quench and Recovery Characteristics of the Zr-Doped (Gd, Y)BCO Coated Conductor Pancake Coils Insulated With Copper and Kapton Tapes", IEEE Trans. Appl. Supercond. Vol. 21, No. 3, pp. 2415-2419, 2011.

[6] O. Tsukamoto, Y. Fu, H. Yoshida, and M. Furuse, "Study on Quench Protection of Coil Wound of Copper or Silver Stabilized YBCO Conductors", Advances in Cryogenic Engineering, Vol. 49A, pp. 823830,2004

[7] Y. Fu, O. Tsukamoto, and M. Furuse, "Study on Quench Protection of HTS Coil Wound of YBCO Conductor", IEEJ Trans. PE Vol. 123, No. 5, pp.661-669, 2003. (in Japanese)

[8] Cryogenics and Superconductivity Society of Japan, Handbook on Cryogenics Engineering and Superconductivity, Ohm Co, Tokyo, 1993. (in Japanese) 